\title{
On the homogeneity of the wave field in coastal regions as determined from ERS-2 and RADARSAT synthetic aperture radar images of the ocean surface*
}

\author{
F. J. OCAMPO-TORRES \\ Departamento de Oceanografía Física, CICESE, Km 107 Carretera Tijuana-Ensenada, Ensenada B. C. 22860, México. \\ E-mail: ocampo@cicese.mx \\ Canada Centre for Remote Sensing, 588 Booth Street, Ottawa, Ontario K1A 0Y7, Canada. \\ E-mail: francisco.ocampo-torres@ccrs.nrcan.gc.ca
}

\begin{abstract}
SUMMARY: Spatial variations of the wave field in coastal waters were determined from images obtained by synthetic aperture radar (SAR) on board the European satellites ERS-1 and 2. The capabilities of RADARSAT SAR to provide useful information for evaluating the wave field variation in nearshore waters are explored. Besides the different polarization between ERS and RADARSAT SARs, range to velocity ratios, signal to noise ratios and the acquisition swath are important issues to take into consideration in comparing the performance of the radar systems. In situ data from a coastal region in the north-west of Baja California are used to validate some of the remote observations and to provide relevant ground truth. Particular aspects of wave phenomena in finite depth waters such as refraction, diffraction and groupiness are considered. An appropriate method for analysing the radar images is applied to describe wave features as they originate from a non-homogeneous process. Wave field characteristics and their spatial variations as resolved by RADARSAT SAR are relevant variables for applications such as beach erosion and coastal management. Inclusion of specific modules to retrieve this type of information should be considered for operational software packages for the use and application of ocean surface data from SAR images. The differences of the two radar systems did not affect their capabilities to observe the wave field in coastal regions.
\end{abstract}

Key words: ocean surface waves, coastal regions, radar images, wave field homogeneity.

\section{INTRODUCTION}

Use of remote sensing data for ocean wave research has mainly focused on deep water conditions with the aim of improving global forecasts of sea-state and of directional wave spectra. Retrieved wave spectra from SAR images have already been compared with global results from forecasting wave models (Heimbach et al., 1998). For the case of nearshore regions, rather few examples of using

\footnotetext{
*Received October 22, 1999. Accepted August 22, 2000.
}

radar information for wave studies have been reported, although there is a great demand for wave data. The environmental and economic importance of sediment transport and erosion on coastal structures is well known. Activities such as navigation, recreation, aquaculture, fishing and others also require a good knowledge of the wave field in coastal regions. Characteristics of synthetic aperture radar (SAR) images are of prime interest for retrieving wave information in shallow water regions. In particular, spatial coverage and resolution render this type of information valuable for determining the spatial 
evolution of the wave field. Effects of bathymetry and currents, among others, enhance the modification of the wave spectrum in the coastal regions, especially over scales of hundreds of metres to a few kilometres. While there is no doubt that SAR images contain wave information, the type of analysis to retrieve it must be carefully considered. The wave field might not be homogeneous (or stationary) and certain characteristics such as wave groupiness, diffraction, and wave focussing might be important and influence the data in such a way that conventional image (or time series) analysis should be applied with caution or should not be used at all. The purpose of the present document is to demonstrate the capabilities of RADARSAT SAR for retrieving information that is valuable for studying in detail some ocean wave characteristics in the nearshore region. Since an important feature of SAR images is their spatial resolution, it will be shown that certain wave field features with scales of hundreds of metres can be measured. The issue of retrieving wave information and applying it to improve forecast systems in the coastal zone through data assimilation will be addressed.

\section{BACKGROUND}

Images of the ocean surface became very popular in 1978 after the mission of Seasat, the first satellite with a synthetic aperture radar on board dedicated to study the global seas (Fu and Holt, 1982).

Subtle spatial variations of the wave spectra were detected by the Seasat SAR off the east coast of North America. While Seasat pass 1339 provided SAR information on two wave systems over a $900 \mathrm{~km}$ strip, a low energy $\left(H_{s} \sim 1 \mathrm{~m}\right) 200 \mathrm{~m}$ wavelength swell was analysed in detail. A set of over one hundred 6.4 by $6.4 \mathrm{~km}$ square frames were processed to yield two-dimensional spectra. However, adjacent spectra were averaged over a $40 \mathrm{~km}$ path to reduce spectral density uncertainty. The wave field had to be assumed to be homogenous for that distance along the satellite path. After correction for the instrument transfer function, the 147 spectra along the path were used to extract some descriptors of the wave field under consideration (wave vector and total image spectral energy). The spatial evolution of the wavenumber was found to be consistent with the expected dispersive behaviour of swell. Sudden changes were observed under the influence of the Gulf Stream, and variations as the waves approach shallow waters were also detected. Spatial variations of wave characteristics were therefore successfully monitored within scales of the order of tens to few hundreds of $\mathrm{km}$ (Beal et al., 1986).

Other remote systems have been used to study particular characteristics and specific aspects of waves near the coast. McGregor et al. (1998) were able to estimate the energy flux of waves propagating over a sand bar by means of a ground-based $\mathrm{S}$ band $(3 \mathrm{GHz})$ Doppler radar. Their measurements resolved the required quantities on scales from metres to tens of metres over a spatial domain of up to $2 \mathrm{~km}$.

Further over the surf zone, some observations have been made with airborne SAR (Shuchman and Meadow, 1980), and derivation of wavenumber spectra and estimation of the dissipation rate of shoaling ocean swell have been achieved by other airborne remote sensors (Hwang et al., 1998).

One of the main applications of radar wave information is in data assimilation to improve wave model forecast on the global oceans (Hasselmann et al., 1997). However, only a few meteorological agencies have been routinely using wave data from remote sensors in their daily forecasting procedures. Furthermore, spectral wave information has only recently been assimilated into global wave forecasting models, since early assimilation methods used only $H_{S}$ to improve model results (Lionello et al., 1992). In order to make the assimilation problem practically feasible in operational systems, only a few spectral parameters characterising the main wave system are typically considered (Dunlap et al., 1998; Hasselmann et al., 1997). In the case of coastal waters, however, the use of radar information to improve local forecasting has been practically non-existent. An exception is the wave model run at the Dutch Meteorological Office for the North Sea (de Valk, 1994), which is not strictly a case of coastal waters, but its dimensions are rather small when compared to the world's oceans. One intention of this work is to encourage the use of wave information retrieved from radar images into data assimilation procedures, since spatial feature information might be of relevance.

Spatial variations of wave spectra have been shown for the northwestern region of the Baja California coast (Ocampo-Torres, 1998). The detailed evolution observed through SAR images was not easy to reproduce with an advance spectral wave model. 


\section{DIFFERENCES IN SAR SYSTEMS AND IMPACT ON THE DETECTION OF WAVE FIELDS}

RADARSAT (ERS) SAR deals with C-band, $5.3 \mathrm{GHz}$, horizontally (vertically) polarised radiation at an altitude of about $800 \mathrm{~km}(780 \mathrm{~km})$. When in Wide Swath Beam mode, it operates with a range to velocity ratio of $\mathrm{R} / \mathrm{V} \sim 120 \mathrm{~s}(\sim 115 \mathrm{~s})$ over a broader acquisition swath than ERS. Larger $\mathrm{R} / \mathrm{V}$ values result in a more non-linear imaging process for viewing ocean waves. This is directly related to an increase in azimuth-travelling wave distortion, associated with the blurring of the detected waves due to the motion of the ocean surface during the imaging process: this is known as velocity bunching. The RADARSAT SAR system is capable of operating in a number of different modes: Standard Beams, Wide Swath Beams, Fine Resolution Beams, ScanSAR Beams, and some Experimental Beams for special purposes. The present work, however, only deals with sea surface images acquired through the Wide Swath Beam mode of operation, which has an appropriate pixel size and resolution to observe ocean surface waves. Image processes from both systems (RADARSAT and ERS-2) produce a pixel size of about $12.5 \mathrm{~m}$ each side. For further details on these modes of operation and on the RADARSAT system, see Parashar et al. (1993), and Luscombe et al. (1993).

\section{IMAGE ANALYSIS AND RESULTS}

While we consider the wave field as a random process, assuming ergodicity and stationarity allows us to apply traditional time series analysis procedures in order to estimate descriptors of the phenomenon under study. In describing the sea surface, the sum of an infinite number of wave components with random distribution of amplitudes, phases and directions of propagation forms the basis. This model for representing ocean waves is very attractive and has long been used in research and application studies. However, the basic assumptions do not hold in many cases in nature. In particular, near the coast the effect of bathymetry, among other factors, enhances some wave-intrinsic features inducing non-homogeneous fields, for instance.

An alternative method of analysis has been suggested (Donelan et al., 1996) in which the use of wavelets permits us to study non-stationary (or non-

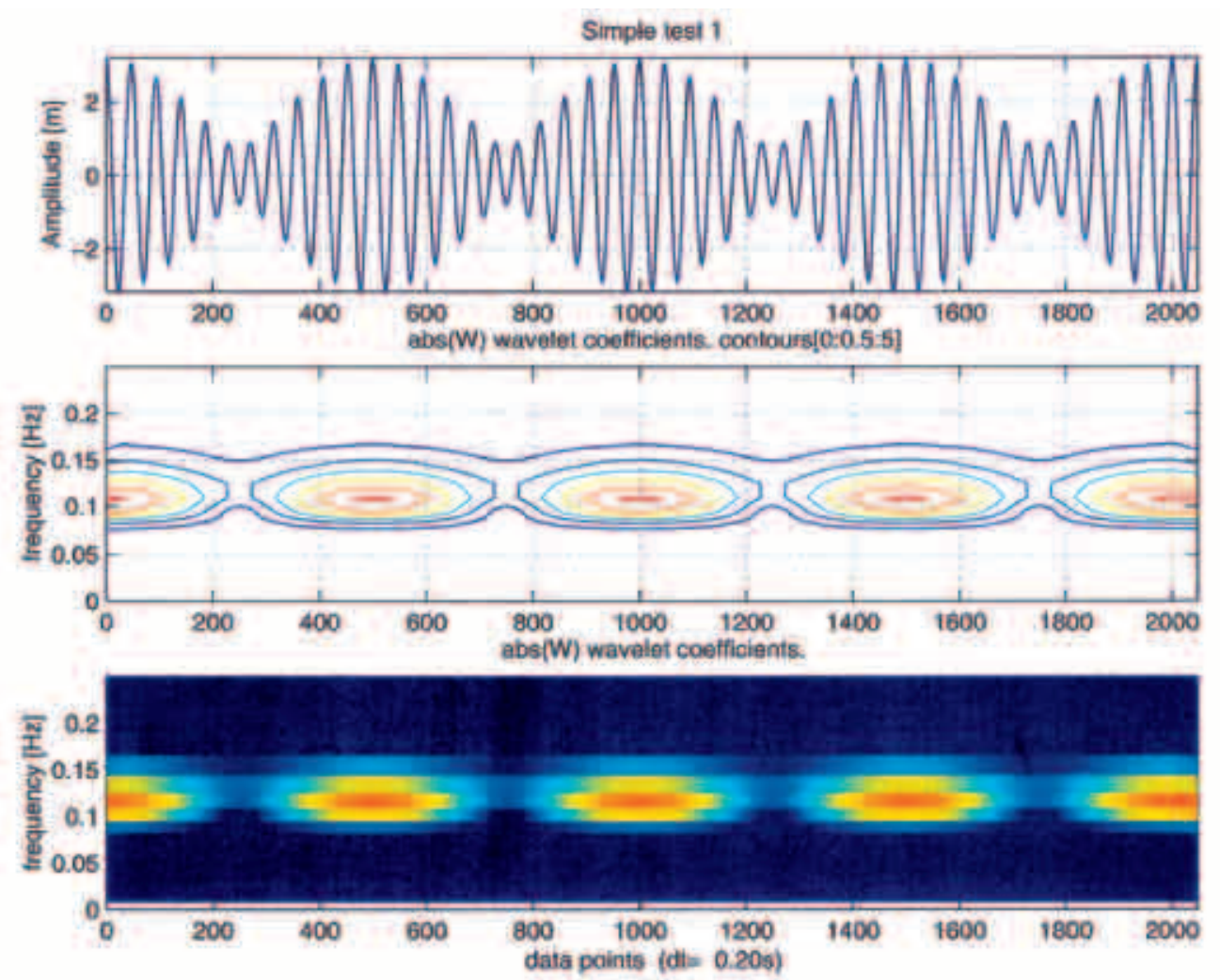

FIG. 1. - Wavelet analysis results from a simple test of adding 2 sine components with amplitude and frequency as $(1.2 \mathrm{~m}, 0.10 \mathrm{~Hz})$ and $(2.0$ $\mathrm{m}, 0.11 \mathrm{~Hz})$ respectively, to reproduce a typical wave record, where a) is the original signal, and the results from wavelet analysis of the original signal are shown as the absolute values of the wavelet coefficients $W_{q p}$ in b) contour and c) colour-coded plots, as a function of time. 
homogenous) processes. Wavelets introduce the idea of representing the sea surface as a superposition of wave groups of varying amplitude, group shape, and group velocity, a concept already put forward by Mollo-Christensen and Ramamonjiarisoa (1978). Wavelet decomposition of a signal allows us to separate the different wave scales (frequencies) present and to look at the variation of their energy as a function of space (time). To perform the analysis in the time domain, a mother wavelet $\psi$ is defined, which can be translated and dilated as

$$
\psi_{q p}(t)=2^{q / 2} \psi\left(2^{q} t-p\right),
$$

where $q$ and $p$ are integers, $p$ being a surrogate for time. Convolving this family of wavelets with a time series $G(t)$ yields the coefficients $W_{q p}$, each providing information about $G(t)$ at the scale $2^{q}$ and time $p$. For more details on wavelet transformation and analysis see Chapron et al. (1995) and Liu et al. (1995).
This method has already been applied in ocean wave research to study sea surface flux and wave modulation due to swell (Peng et al., 1995) and to determine some characteristics of wind stress over groups of waves (Liu et al., 1995).

In order to illustrate some of the basics of this method, a simple exercise was run. A sum of two sine waves with similar frequencies $\left(f_{1}=0.10 \mathrm{~Hz}\right.$, $f_{2}=0.11 \mathrm{~Hz}$ ) is taken as the original signal representing wave groups in a simple fashion (Fig. 1a). The analysis through the wavelet method shows the distribution of energy levels as a function of the spatial position. As an average, the highest energy is concentrated at the average frequency of the two components. This energy, however, varies with the distance, as a result of the wave groupiness. From this very regular wave pattern, the change in energy as a function of space is also very regular, and closely follows the envelope of the wave profile. The absolute value of the wavelet coefficients is shown

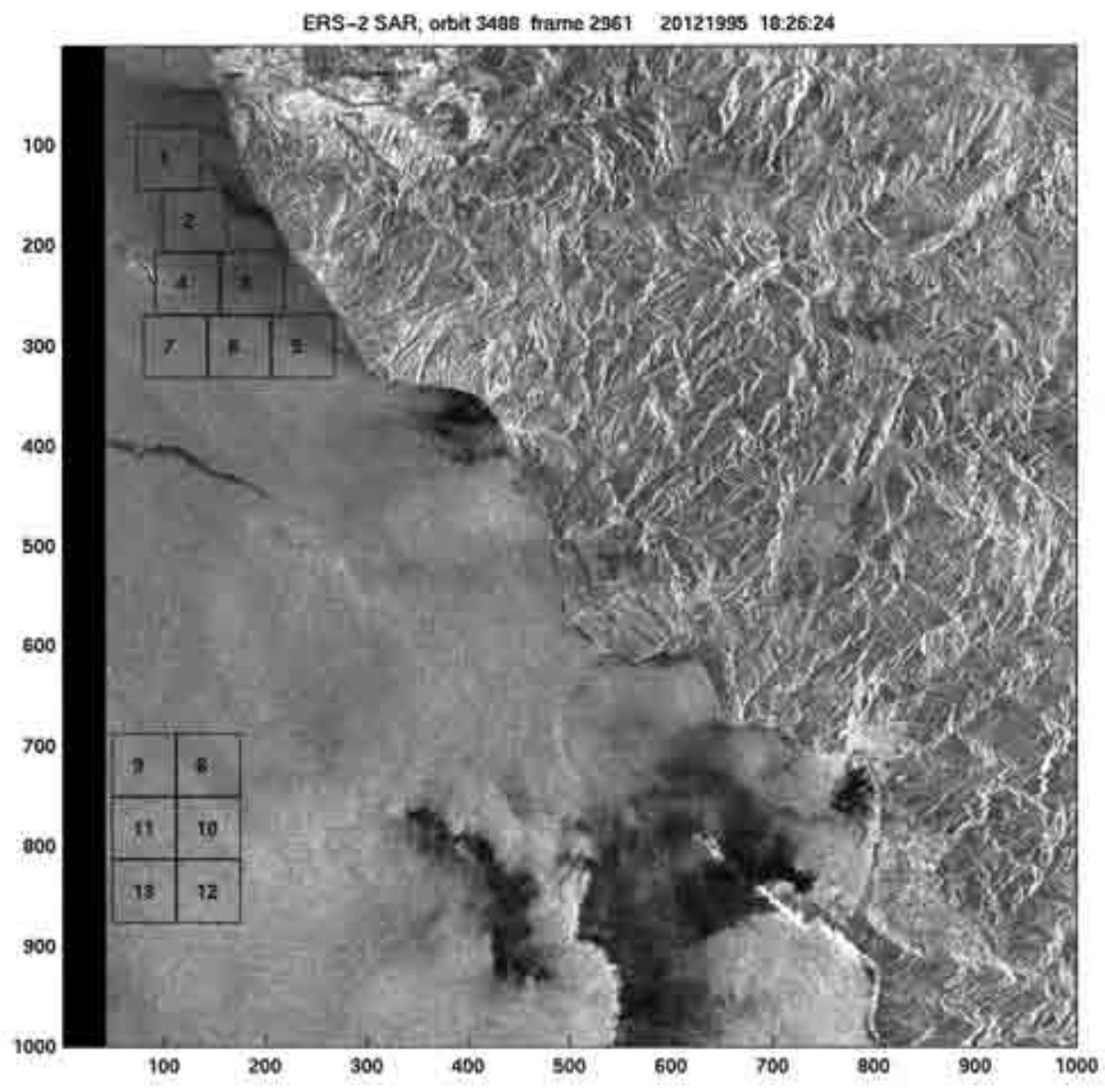

FIG. 2. - ERS-2 SAR image (orbit 3488, frame 2961) for the north-west coast of Baja California acquired by Norman Station on December 20,1995 at $18: 26$ UTC. Coordinates for the image centre are $32.07^{\circ} \mathrm{N}$ and $116.88^{\circ} \mathrm{W}$. The coastline is well depicted and the 13 subimage regions for spectral calculations are shown. Subimages 1-7 are for the shallow water region between Coronado Islands and Rosarito Beach, while 8-13 are for the deep water case. Dimensions of the image are $100 \mathrm{~km}$ by $100 \mathrm{~km}$. Each subimage is 512 by 512 pixels $(6.4 \mathrm{~km}$ by 6.4 km approximately). (C) ESA 1995. 
in Figs. $1 \mathrm{~b}$ and 1c, which present the same information with contour and colour-coded types of graphs respectively.

\section{Example of ERS SAR images}

Some ERS SAR images were obtained for the region of study under the project Wind, Waves and Mixing in the Coastal Zone, supported by the European Space Agency. Figure 2 shows as an example (8-by-8 average scene), an image acquired on December 12, 1995, at 18:26:24. The area of interest, in the upper-left corner of this figure, has a relatively wide continental shelf and includes Coronado Islands (left of Sub-image 4) and the nearby coastal zone in front of Rosarito Beach. The bright region at the northernmost part, close to the coastline, is the City of Tijuana. In the lower part of the image, Todos Santos Bay can be seen. We can also see some interesting features on the ocean surface that might be related to ocean and/or atmospheric fronts, the presence of surfactants, and calm sea on the lee of coastal points or capes.

Full resolution sub-images were extracted from the original image for further analysis. By inspection, we can see variations in the wave field. Furthermore, through spectral analysis, we can estimate detailed variations in the wave characteristics in the nearshore region along the coast. Spatial variations in these spectra can also be observed. Key parameters such as the relative variation of spectral peak wavelength and significant wave height can be obtained along the coast (Ocampo-Torres et al., 1997). These variations can also be considered as evidence of the non-homogeneity of the wave field we are dealing with. However, the analysis in this case focuse on the spectral shape and its variation in the nearshore region.

Results from spectral analysis for both deep and shallow water wave cases are shown in Figure 3. In order to characterise the deep water wave field, image spectra are estimated from Sub-images 8 to 13. Trend removal, cosine window tapering, 2-by-2 pixel smoothing, 6 (512-by-512) sub-image spectra averaging, and averaging 4 adjacent bands results in spectral estimates with 48 degrees-of-freedom. The energy spectrum as a function of wavenumber $\left(K_{x}\right.$ in range, and $K_{y}$ in azimuth) is illustrated in Figure 3 a. The dotted circles represent the loci for wave components with $400 \mathrm{~m}, 200 \mathrm{~m}$ and $100 \mathrm{~m}$ wavelengths. Spectral peak components have approximately a $300 \mathrm{~m}$ wavelength propagating towards about $115^{\circ}$ (the satellite path was about $10^{\circ}$ from North). The wave direction deviated by only $15^{\circ}$ from radar range direction (along the $K_{x}$ axis, or the horizontal co-ordinate in the image). This wave field is a very well-defined swell, with a characteristic direction and components with wavelengths varying from 400 $\mathrm{m}$ to $200 \mathrm{~m}$. A spectral tail with low and shorter wave components can also be seen in this spectrum. Of course, it is assumed that the wave field is homogeneous in this deep water region, in order to apply the FFT procedure and the averaging of the several sub-image spectra.

Spectra were obtained from sub-images numbered from 1 to 7 in the image. Each sub-image is 512 by 512 pixels, similar to those extracted from the deep water region. From the image spectrum shown in Figure 3b, it can be observed that the wave field within the continental shelf is already affected by at least the refraction process. The waves mainly propagate along the radar range direction. A secondary peak is noticed, representing wave components with approximately $180 \mathrm{~m}$ length. As the wave field propagates towards the coast, shallow water processes continue to act. The mean wave direction turns slightly north (Fig. 3c) and further enhancement of even shorter waves is noticed as the secondary peak is now shifted towards slightly higher wavenumbers. Near the coast, it seems that only waves slightly longer than $200 \mathrm{~m}$ survived, as can be seen in Figure 3d. However, the wave field was decomposed into two wave trains travelling about $10^{\circ}$ and $50^{\circ}$ north from the range direction. Depending on the directional resolving power of the measuring device, this could indicate some broadening of the wave spectrum.

\section{RADARSAT SAR images for the NW region of Baja California}

Four RADARSAT SAR images were acquired over the northwestern region of Baja California, Mexico, on December 19, 1998, January 12, and 26, and on July 20, 1999 respectively. Some in situ measurements were carried out in the study area. Wind information and significant wave height from a meteorological buoy are available for all four images dates. Typical light-to-moderate wind conditions were recorded during image acquisition times, while significant wave heights of approximately $1 \mathrm{~m}$ and close to $2 \mathrm{~m}$ were measured on December 19, and January 26 respectively. The location for the instruments deployed and some bathymetric information 
a

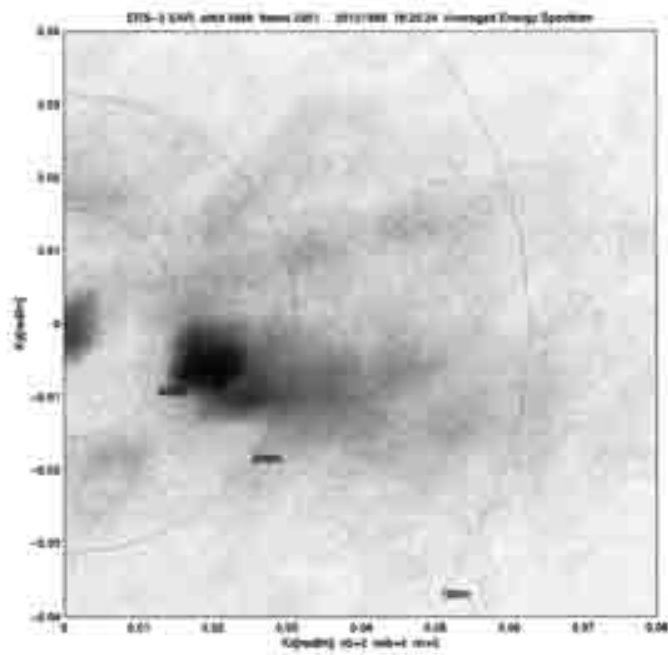

b

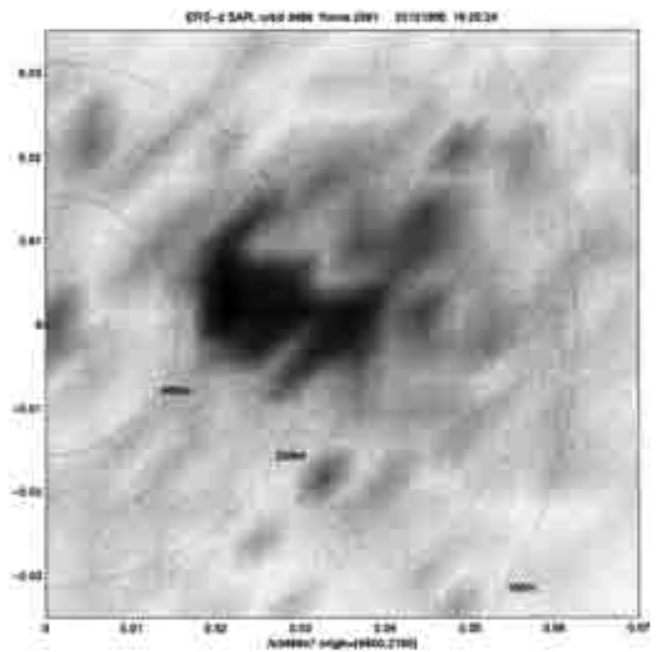

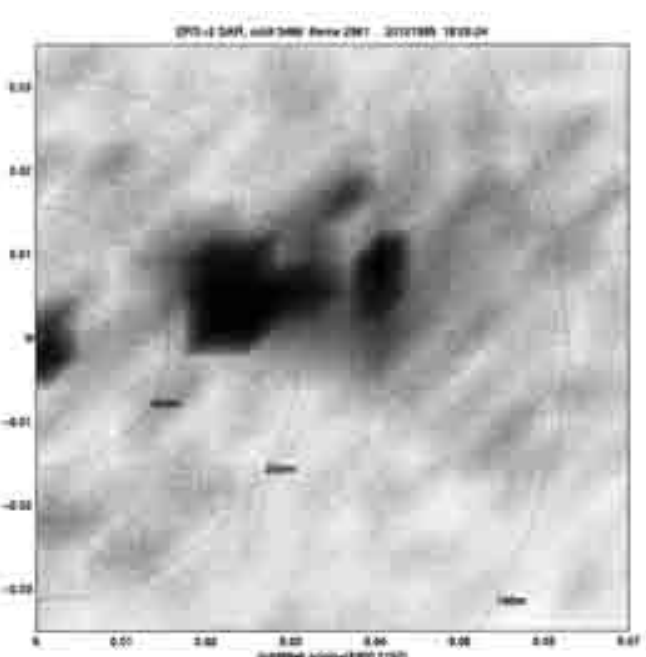

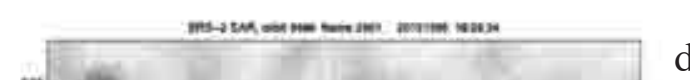

d

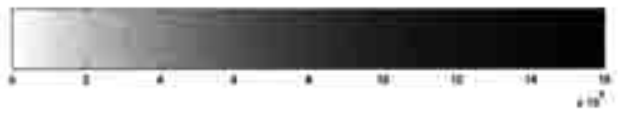

FIG. 3. - Estimated image spectra from some subimages shown in Figure 2. For deep water waves, case a) subimages 8-13, and for shallow to intermediate water waves cases b), subimage 7, c) subimage 6 , and d) subimage 5 . Grey tones are for spectral energy density as a function of the wavenumbers $K_{x}$ and $K_{y}$ in range and azimuth direction respectively. Dotted circles are the loci for $400 \mathrm{~m}, 200 \mathrm{~m}$, and $100 \mathrm{~m}$ wave lengths.

on the study area are shown in Figure 4. These measurements were obtained as part of some field campaigns of the project Ocean Waves in Coastal Regions (supported by CONACyT, México), with the aim of studying wave spectral evolution and preparing the area as a long term instrumented site for wave research and calibration/validation experiments for future satellite remote sensing missions.

Hourly records of wave elevation and orbital velocity were obtained with a SeaPack2000, (pressure transducer and electromagnetic current sensor) from Woods Hole Instruments Ltd. Measurements at $2 \mathrm{~Hz}$ and the sensor characteristics (Paroscientific Quartz Pressure sensor, with 0 to 100 psi range,
$1.0 \mathrm{~cm}$ precision, and $0.1 \mathrm{~cm}$ resolution) provided us with a confident estimation of the wave parameters. Results from these measurements have shown that spectral peaks were centred at about $0.0815 \mathrm{~Hz}$ and $0.0737 \mathrm{~Hz}$ before and after image acquisition. Corresponding wavelengths of main wave fields for this region where the sensor was located at about $18 \mathrm{~m}$ below mean water level are $168 \mathrm{~m}$ and $149 \mathrm{~m}$ respectively.

Figure 5 shows the RADARSAT image acquired on December 19, 1998. Contrast and brightness has been adjusted to enhance sea surface features, while land information is saturated and essentially masked. Although there is no prominent wave sig- 


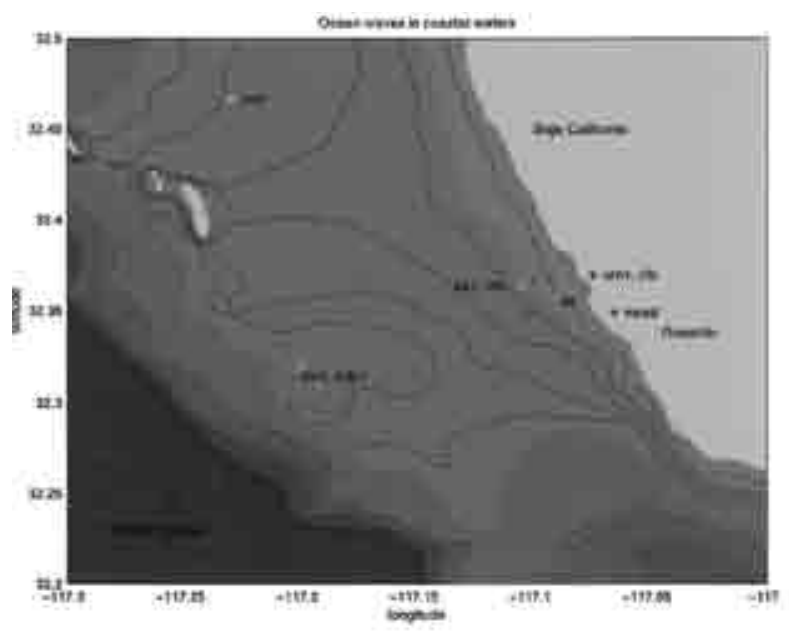

FIG. 4. - Coastal area in the north-west of Baja California, where some in situ measurements are being carried out. Coastal meteorological buoy aa1, directional wave and current sensors whi and s4, and marine radar mrad have been in quasi-continuous operation since January 1998. A meteorological buoy $\mathbf{m m} \mathbf{1}$ operated for over a month in early 1998. A directional wave buoy dw1, a Doppler current profiler adp1 and a coastal meteorological station em1 are planned to be installed. Bathymetric contours are 10, 20, 30, 40, 50, 100,200 , and $300 \mathrm{~m}$. nature, other interesting characteristics are observed over the ocean, such as the cloud-like pattern along the left edge of the image. The dark areas near the coast might be related to localised regions that are sheltered from the wind.

The scene taken on January 12 is shown in Figure 6 as a full-size image (Fig. 6a) and an enlargement of the study area (Fig. 6b). Again, large-scale oceanic features are easily observed, and in this particular case a dark irregular stripe running northsouth attracts attention. This helps to stress the point that local scale variation of many phenomena can arise on the ocean surface. Figure $6 \mathrm{~b}$ is a typical example of non-homogeneous wave features in the coastal zone detected through SAR imaging process.

A closer look at the shallow waters reveals a complex combination of wave patterns and sea surface slicks (irregular streaks). Wave diffraction is noticeable, as is the refraction process acting as the waves approach the coast. Very close to the coast-

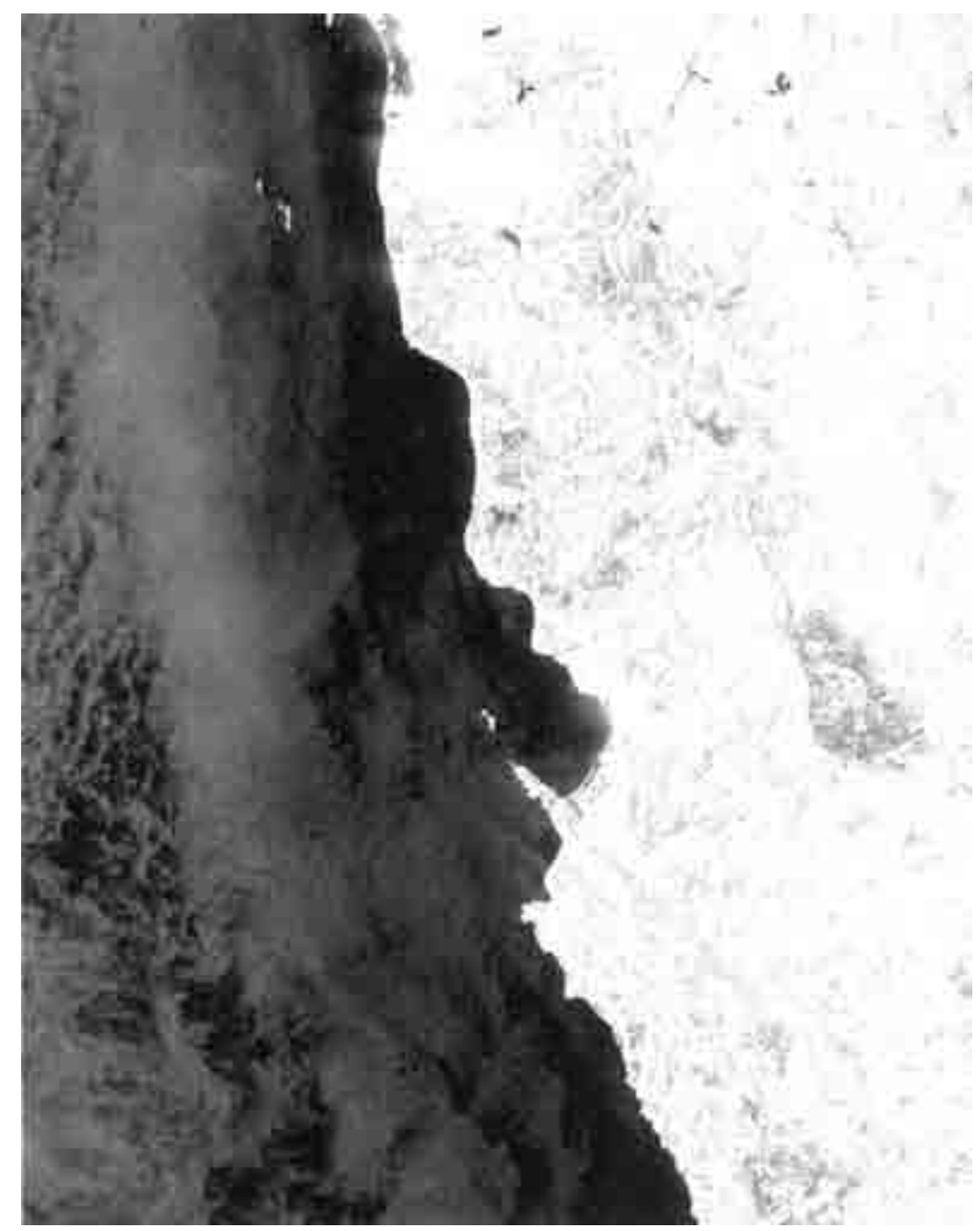

FIG. 5. - RADARSAT SAR image acquired on December 19, 1998, at 01:47:168 UTC, showing the northwestern region of the Baja California Peninsula, México. Wide Swath Beam 2 mode was used to cover an approximate area of $138 \mathrm{~km}$ (range) by $178 \mathrm{~km}$ (azimuth). Pixel size is $12.5 \mathrm{~m}$ by $12.5 \mathrm{~m}$, although this picture is resolution degraded through application of a 16-by-16 average filter. (C) CSA 1998 . 

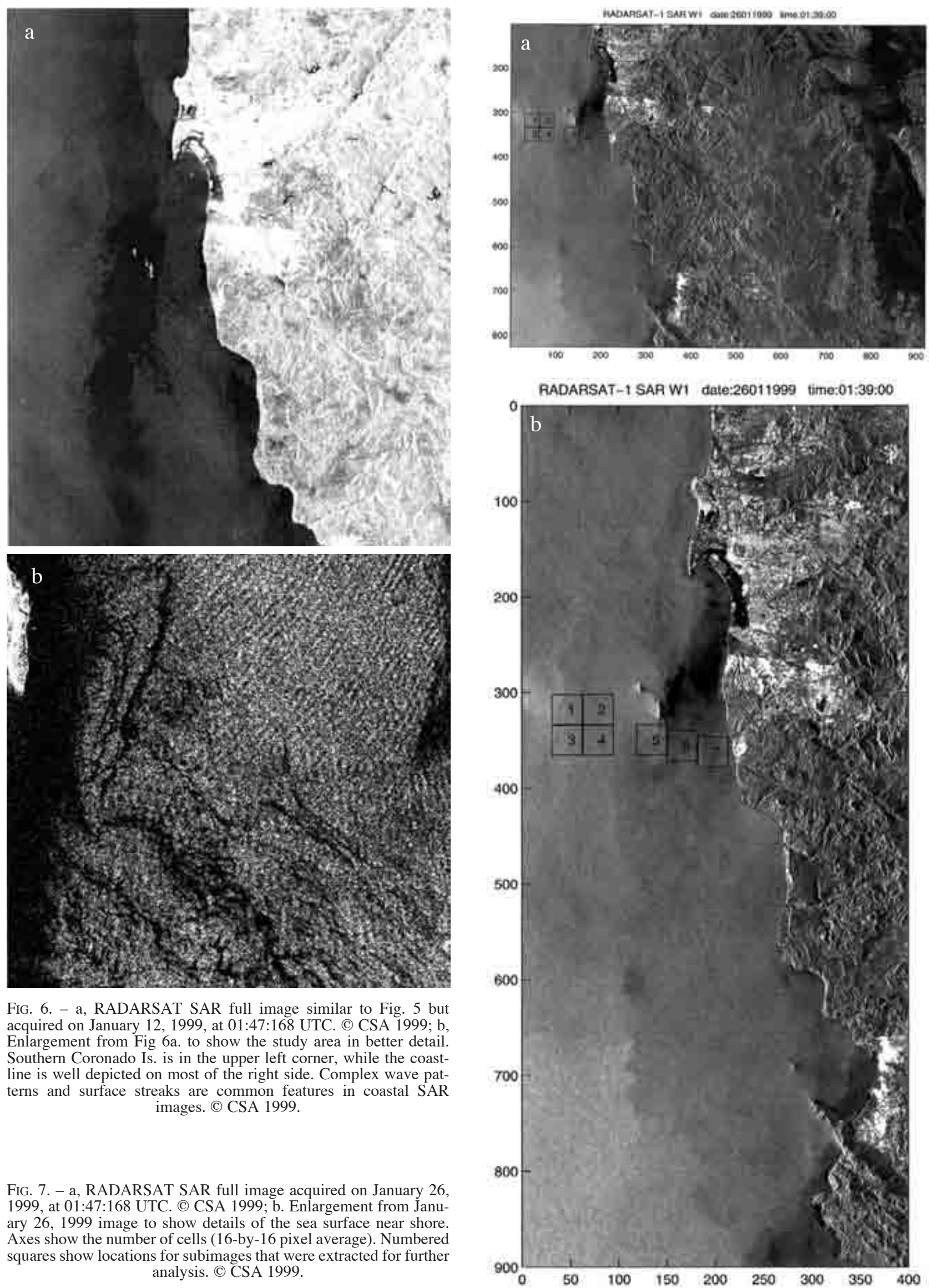

FIG. 7. - a, RADARSAT SAR full image acquired on January 26 , 1999, at 01:47:168 UTC. (C) CSA 1999; b. Enlargement from January 26, 1999 image to show details of the sea surface near shore. Axes show the number of cells (16-by-16 pixel average). Numbered squares show locations for subimages that were extracted for further analysis. (c) CSA 1999. 
line, wave crests can be observed as they are packed together because wavelengths are shortened. Wave crest orientation (relative to the coastline) and wavelength are important characteristics that influence the erosion/deposition rate on the sandy beaches of the region. It is obvious that these characteristics may change from point to point along the coast. This variation can be monitored by information obtained from SAR images. Furthermore, this type of information can also be obtained for extreme waves reaching the coast, such as those generated by intense storms or hurricanes. The benefits of having access to this type of information are numerous.

The full image acquired at 01:39 on January 26, 1999 is shown in Figure 7a, while in Figure 7b shows an enlargement of the coastal region. An interesting feature is the large-scale changes in backscattered level, caused by variations in the atmospheric boundary layer. Darker zones, for example, between Coronado Is. and the Tijuana coastline, might be associated with surface slicks. A rather large brighter zone in the lower left corner, off Todos Santos Bay, can be attributed to differences in surface waters and/or lower atmospheric conditions in the area.

Sub-images 1 to 7 were extracted for detailed analysis. The imaged wave field can be analysed in detail from these sub-images. In particular, it is observed that there is a dominant wave field travelling practically in the range direction, towards the coast. Sub-images were therefore extracted along that direction in order to determine some aspects related to the spectral evolution as the waves approach the coast. Figures $8 \mathrm{a}$ and $8 \mathrm{~b}$ illustrate the change in the wave field from offshore to the nearshore region. It is clearly seen that the main wave pattern in Sub-image 3 (Fig. 8a) has shorter wavelength scales than that in Sub-image 7 (Fig 8b). This variation is easy to notice and it is not difficult to assume that the spectra will also show these differences. The wave field offshore might be thought of as representing a homogeneous process, at least in the sub-image space domain $(\sim 6.4 \mathrm{~km})$. However, this is not an obvious assumption to make on the wave field near the shore.

\section{Wave spatial variations estimated through wavelet analysis}

A section of the January 26 image between Coronado Is. and Rosarito Beach is shown in Figure 9, where four along-range transects were extracted. Wavelet analysis was applied to these four 1024 pixel
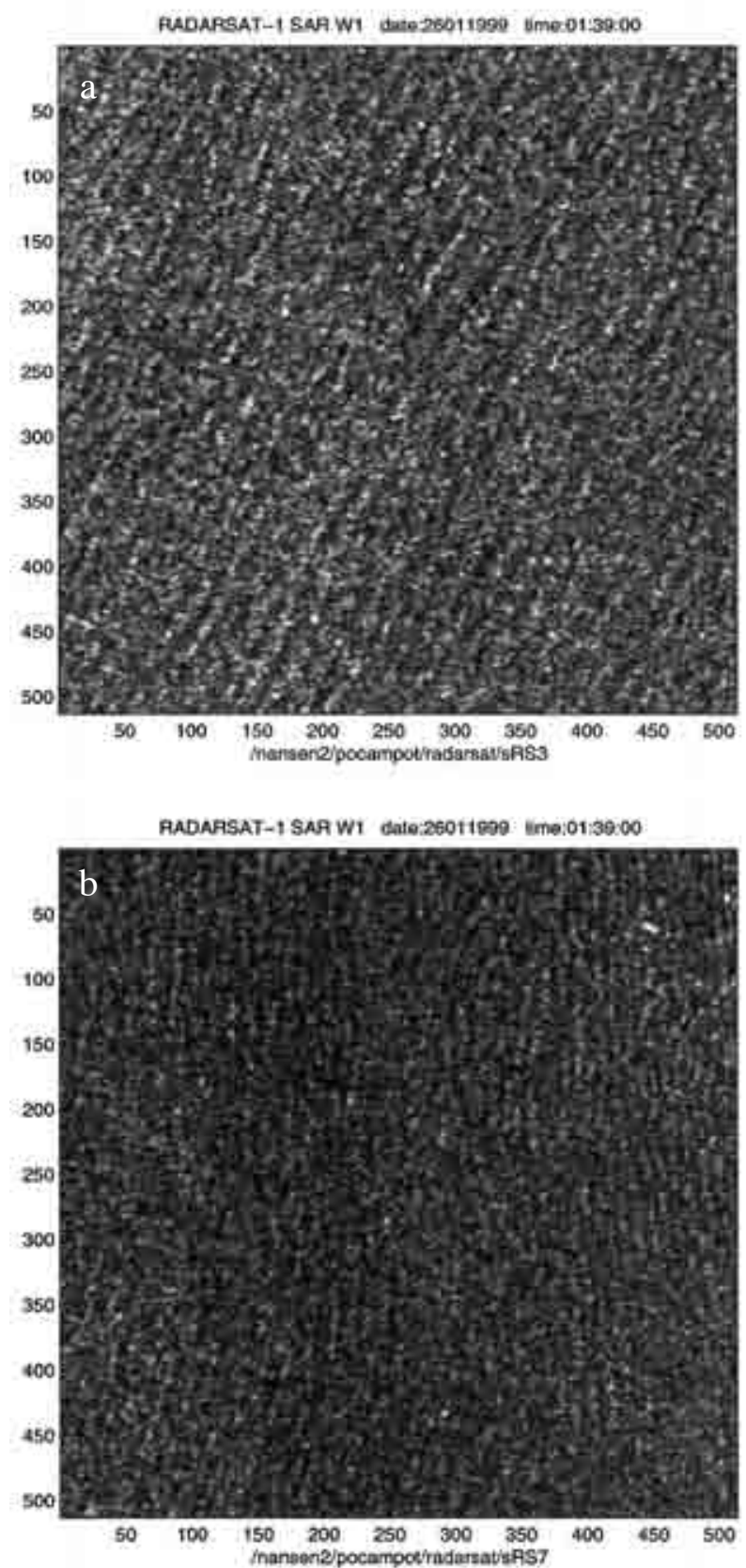

FIG. 8. - a, Enlargement for Subimage 3 from Figure $7 b$ to show the wave field offshore; $b$, Enlargement for Subimage 7 from image $7 b$ to show the wave field in the near shore region. Axes numbers are pixels. C) CSA 1999.

transects in order to determine the energy distribution as a function of wave scale towards the coast. It is important to notice that the waves are practically range-travelling over this area, allowing us to deal with a relatively linear wave imaging process.

Another readily noticeable feature in Figure 9 is a curved border between bright and rather darker zones south-east of the southern tip of Coronado Is. This is well correlated with the bathymetry contours seen in Figure 4. 


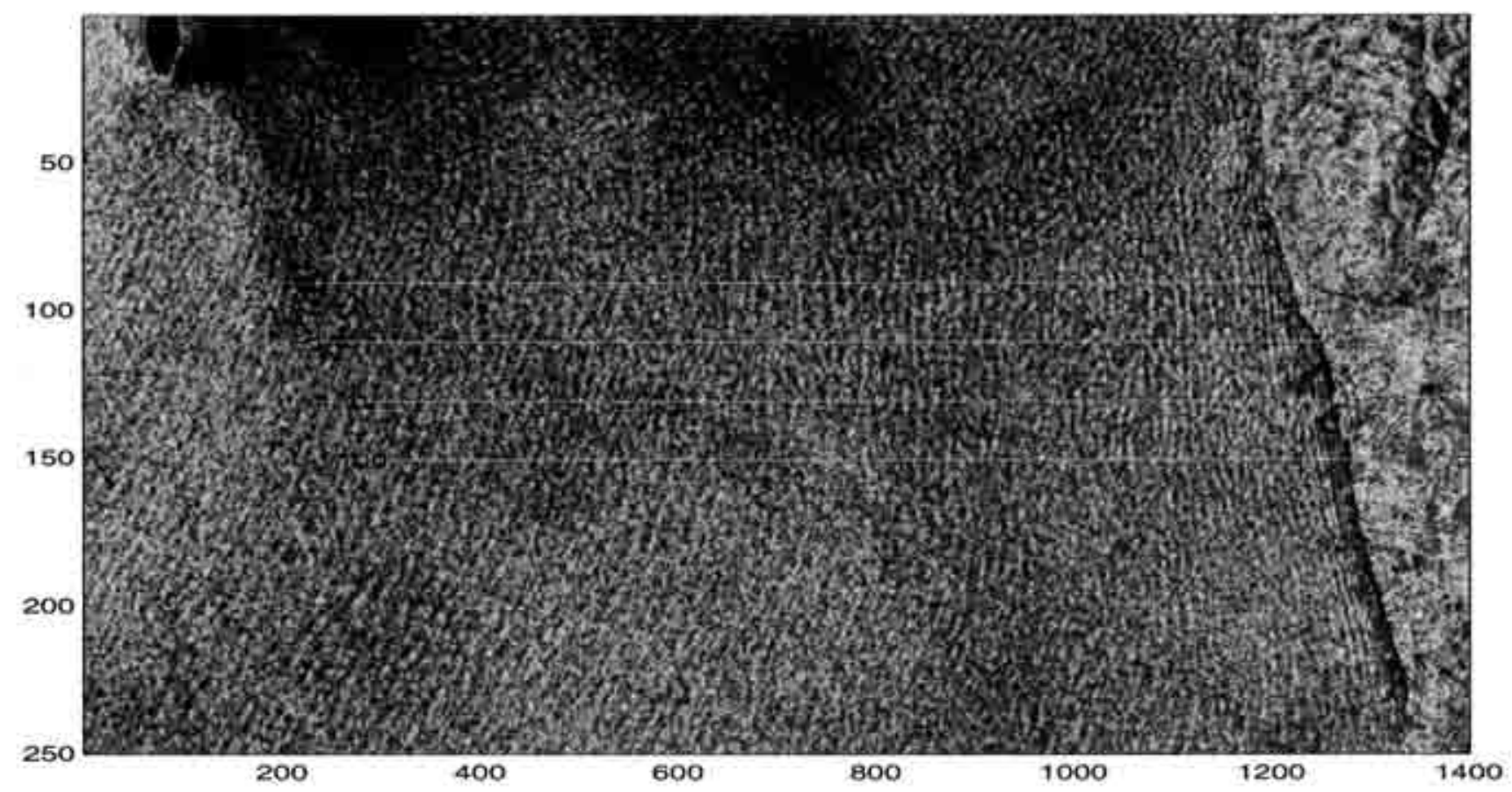

FIG. 9. - Enlargement of the coastal section from the image acquired on January 26, 1999, showing four range transects extracted for wavelet analysis. Coronado Is. is in the upper left corner and the four 1024 pixel transects are offshore from Rosarito Beach. (C) CSA 1999.

A diffraction pattern is also detected, as the island acts as an obstacle to incoming waves from the Northwest. Cross wave patterns can even be observed in most of the upper part of this scene (Fig. 9), between the islands and the coastline. This type of complex wave pattern with at least two wave trains with different directions of travel is not an uncommon feature in this Pacific coastal region. This is of particular interest when is considered in the description of the wave field spectral terms, since none of the present wave spectral models include diffraction processes in their numeric schemes.

Some results obtained from wavelet decomposition applied to the transects shown are presented in Figures 10 to 13. Each 1024 point transect represents a 4-row bin averaged signal. The purpose of this averaging is to reduce noise and increase statistical confidence in the estimated wavelet coefficients. In each case, the upper panel shows the signal once the mean is removed. The wavelet coefficients are estimated over the scale range $0.001 \mathrm{~m}^{-1}$ (wavelength, $\lambda=1000 \mathrm{~m})$ to $0.0287 \mathrm{~m}^{-1}(\lambda \sim 30 \mathrm{~m})$, over 40 bands in total. The centre and lower panels show the absolute value of the wavelet coefficients $W_{q p}$, as a function of distance towards shore (given as the number of pixels), as contour and colourcoded plots, respectively. Since the pixel size is 12.5 $\mathrm{m}$, each transect is about $12.8 \mathrm{~km}$ long. The most energetic scale was $0.004 \mathrm{~m}^{-1}$ for the lower transect (label 100), and varied to $0.007 \mathrm{~m}^{-1}, 0.009 \mathrm{~m}^{-1}$ and $0.006 \mathrm{~m}^{-1}$ for the other three, labelled 120, 140 and 160 respectively. In average terms, wavelengths between $240 \mathrm{~m}$ and $110 \mathrm{~m}$ contained the most energy in this sector of the scene. This energy within these most energetic bands, however, varies considerably with distance towards the coast, as evidence of the groupy nature of wave phenomenon. It is, in fact, possible to detect the presence of high energy within the main wave scales with some repeatability. In the results from the first transect, for instance (Fig. 10), there is an enhancement of energy every 120 to 140 data points, equivalent to 1500 to $1750 \mathrm{~m}$ approximately (in the order of 6 to 7, $240 \mathrm{~m}$ waves or 10 to $11,160 \mathrm{~m}$ waves). The occurrence of energy enhancement is repeated with a closer spacing as the water gets shallower approaching the coast.

The spectral shape evolution is also a peculiar characteristic of these results. It is easy to observe that the wave spectrum in some areas is very narrow (most energy contained in very few wave scales), while it widens considerably in some others. A tendency to shift the main energy towards the shorter wave scales is consistent with the shortening of waves as they propagate into water of decreasing depth. 

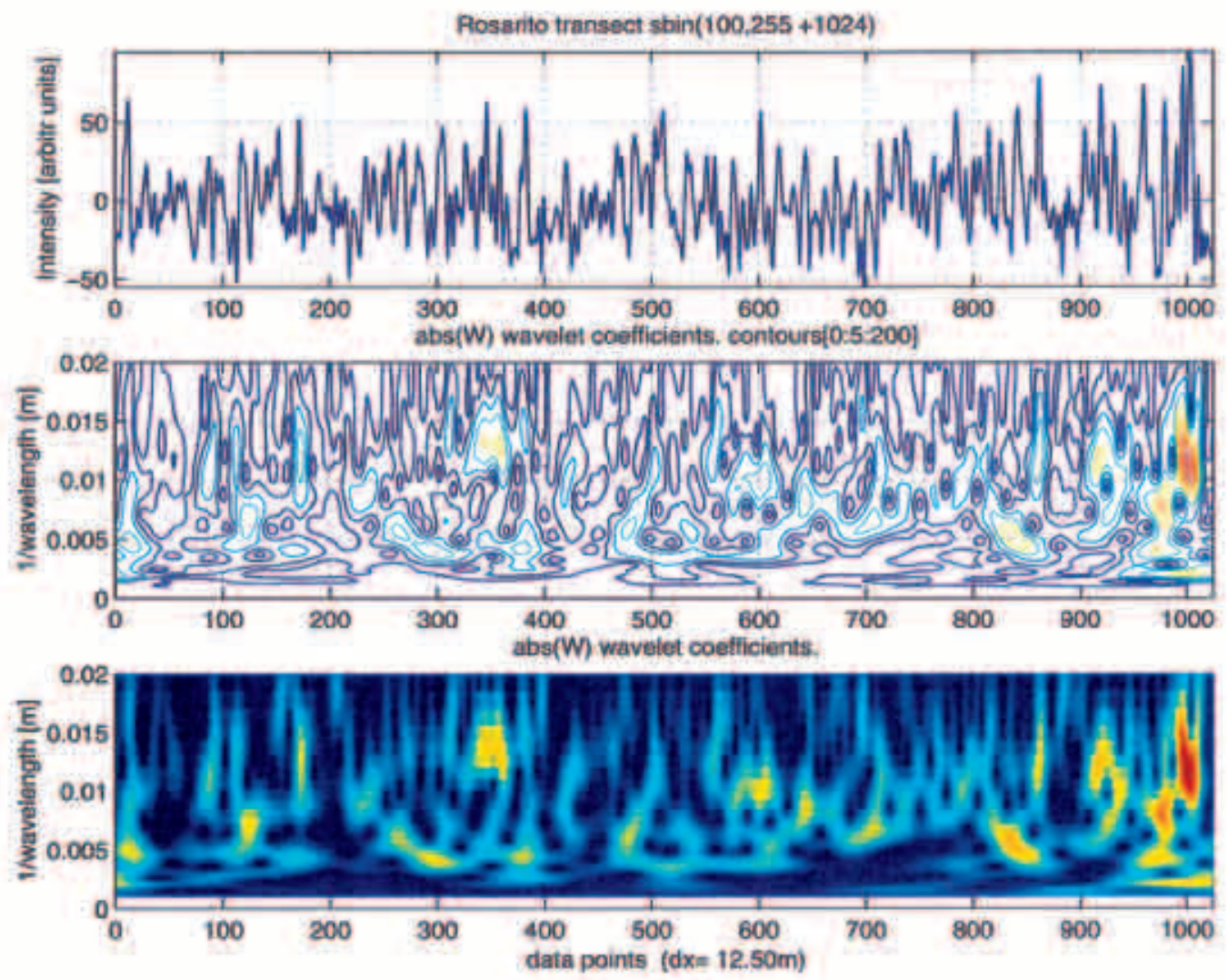

FIG. 10. - Results from applying wavelet analysis to the lower transect (label 100) in Figure 9. The upper panel shows the original signal, a 4-row averaged transect as a function of distance towards shore (in pixel number). The centre and lower panels show the wavelet coefficients representing the energy distribution over the wave scales (vertical axis) resolved as a function of the distance along the transect.
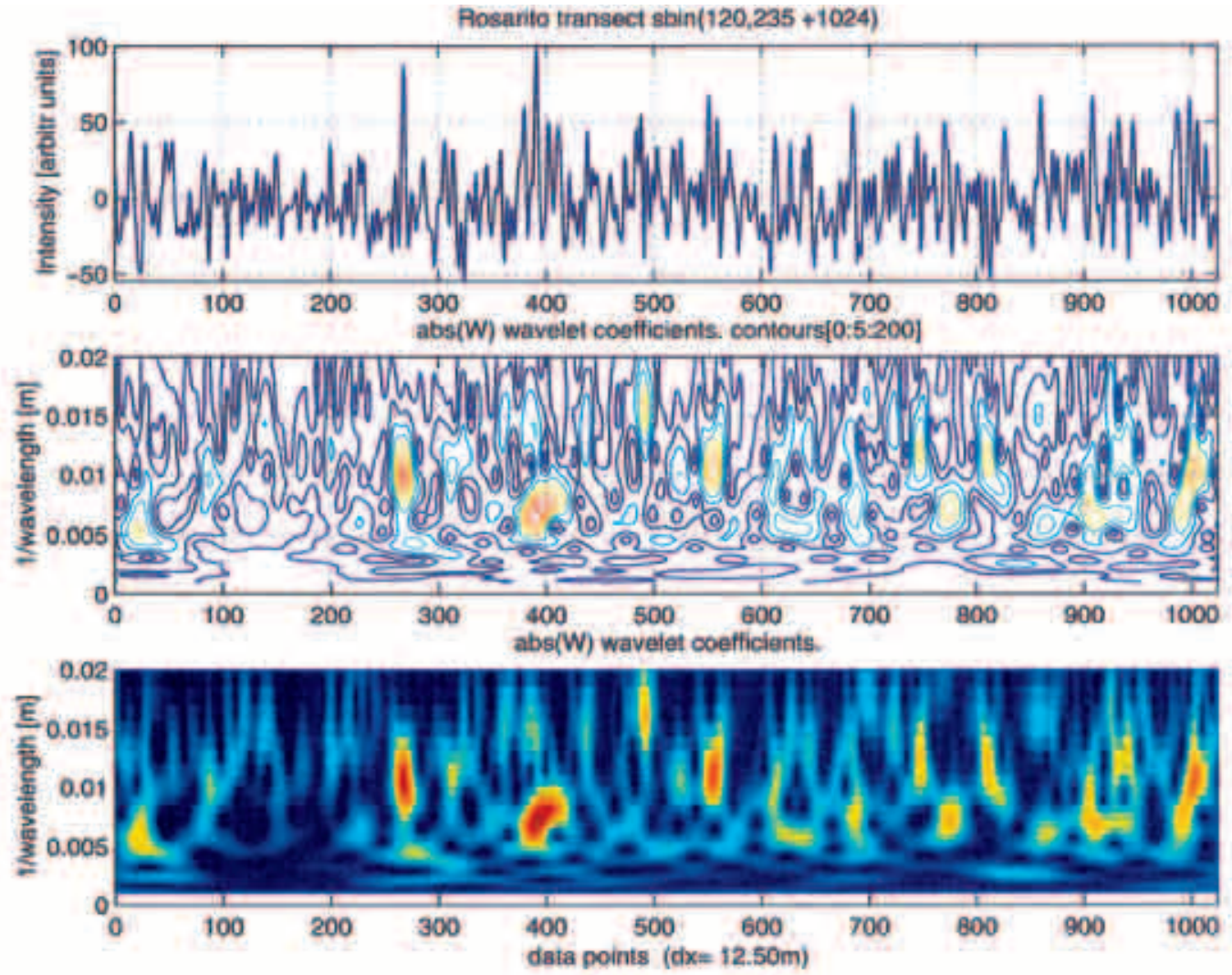

FIG. 11. - Same as Figure 10, but for the second transect (label 120). 


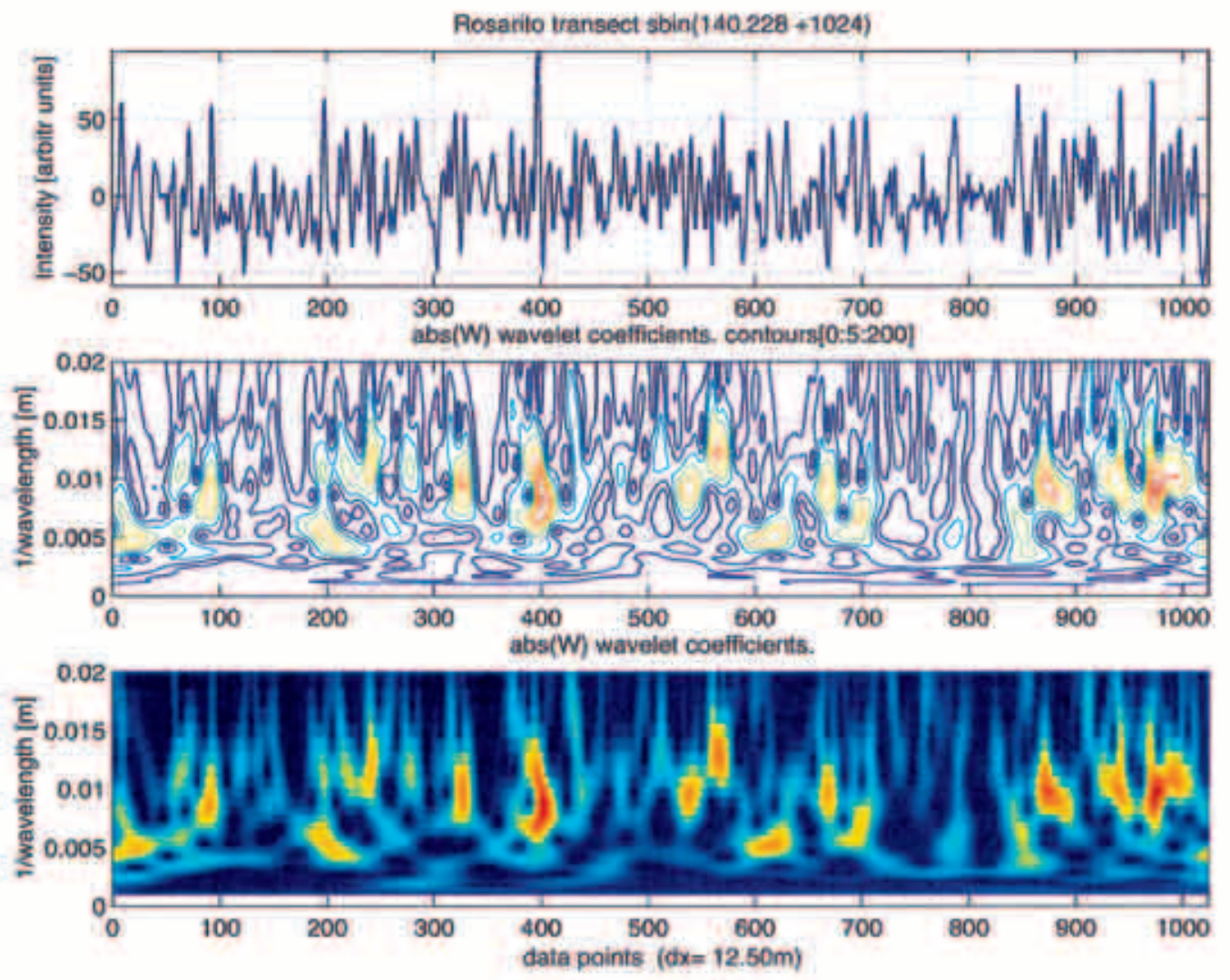

FIG. 12. - Same as Figure 10, but for the third transect (label 140).
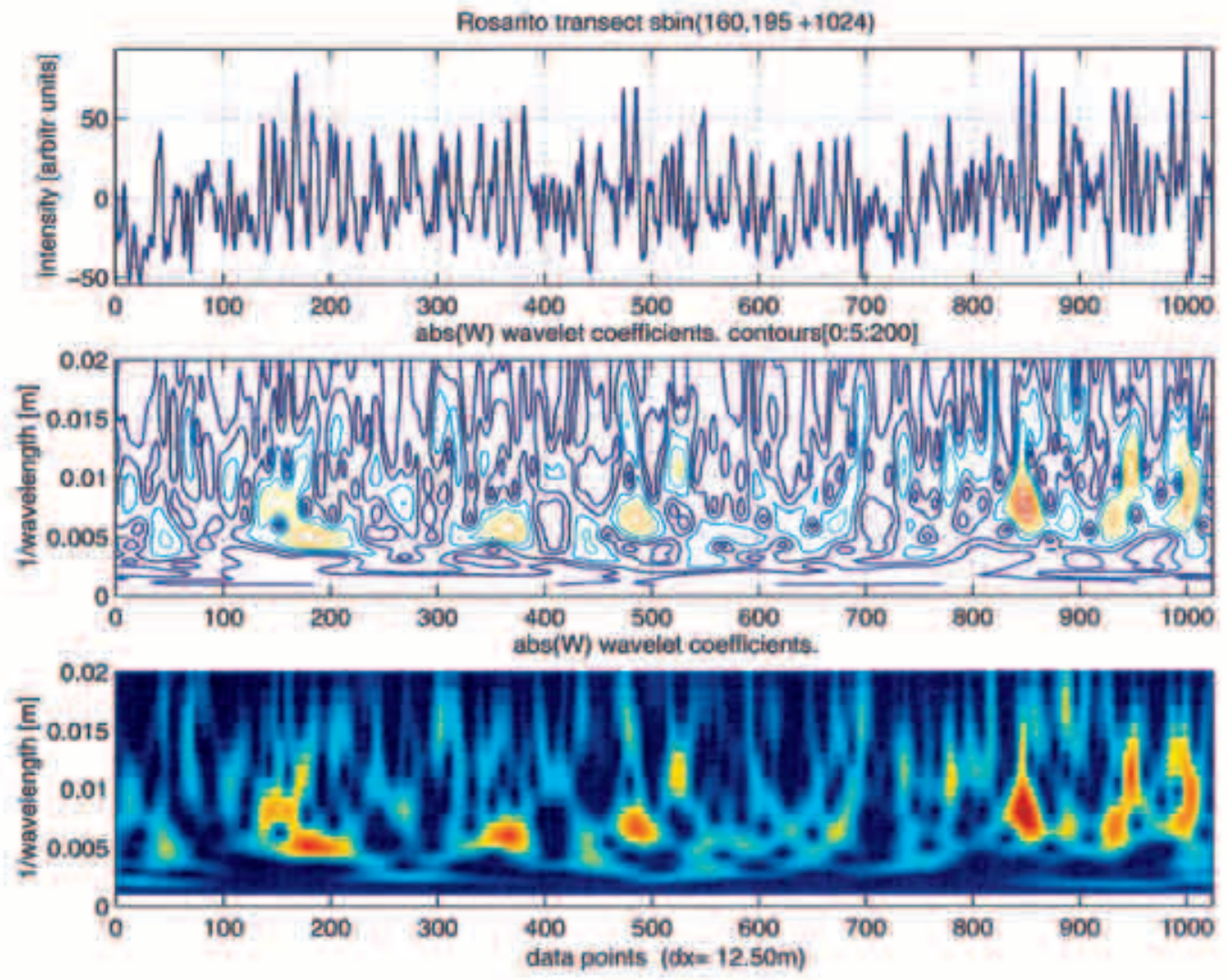

FIG. 13. - Same as Figure 10, but for the top transect (label 160). 


\section{CONCLUSIONS AND RECOMMENDATIONS}

Spatial resolution and coverage by RADARSAT SAR are very important characteristics providing us with the ability of look at the wave field near the coast in a rather unique fashion, and to achieve a detailed description the evolution of wave as they approach the coast. Both spatial resolution and coverage are key issues in allowing the use of SAR images to study local effects in the wave field and to deliver crucial information on coastal applications such as beach erosion and mixing processes in the near shorezone. Spatial variability of the wave field should be carefully taken into account when wave information retrieved from radar images is compared with the estimates from wave records in the time domain. The relevance of wave information from radar images is such that wave retrieval modules should typically be included in application packages for the use of ocean surface SAR images. End users should benefit by improved management of coastal/marine operations, beach erosion, and through compilation of needed wave data for long term statistics and climatology.

Although further analysis is recommended, especially for full inversion of the image information to retrieve ocean wave spectra, wave characteristics are shown to be determined directly from the images and from the processing carried out. In particular, the fact that RADARSAT SAR uses HH polarization does not seem to have a negative effect in comparison with ERS which uses VV polarization, on its ability to observe the ocean wave field in the area of study. It has already been established that a large range to velocity ratio results in SAR image spectra that are more susceptible to non-linear imaging processes and azimuth spectral cut-off (Vachon, et al, 1994). The small differences in the R/V parameter between the ERS and RADARSAT SAR systems, however, shows no noticeable effect on their abillity to detect wave features in the coastal zone with the desired spatial detail.

Particular features of the wave phenomenon are easily detected through SAR images, such as diffraction, refraction and groupiness. These are important issues that characterise wave evolution in intermediate to shallow water depth regions. Very close to the coastline, where the waves are close to breaking, the crests may be easily detected. Through a proper analysis that deals with non-homogeneous processes, the distribution of wave energy over the various wave scales present can be described as a spatial function as the waves approach the coast. The wave energy has been shown to vary substan- tially with approach to the coastline, enhancing the groupy nature of the ocean waves.

Information from extreme events is very valuable because they could be the main cause of disasters, not only within the coastal zones, but also offshore. Radar information on the wave field under intense storms or hurricanes might help to improve sea-state and atmospheric forecasting models.

Extending the analysis of the wave field as a nonhomogeneous process is a natural step forward. Two-dimensional wavelet decomposition should be implemented and applied to SAR images of waves in nearshore regions.

In view of future space-borne SAR missions, it is important to stress the fact that wave information retrieved from SAR images might be very valuable for numerical forecasting procedures through data assimilation schemes, with special emphasis on local to regional scale phenomena. Of course, an enhanced resolution to accurately describe coastal phenomena is needed, which might be achieved by nesting finer into coarser resolution models. Although the frequency of coverage of a determined coastal area is relatively low, the development of light spacecraft must be important in a future constellation of SAR systems to monitor the ocean. Spatial and temporal variability should, however, be better resolved, observed in more detail, and studied further to include them suitable in data assimilation schemes.

\section{ACKNOWLEDGEMENTS}

This work was supported by CONACyT, Mexico through project 225080-5-4302PT, accelerated initiative Fase 1 de Oceanografía por Satélite (DAJ J002/750/00) and a sabbatical grant, and by SIMAC (970106002). ERS SAR images were provided by ESA under project Wind, Waves, and Mixing in Coastal Regions (ESA AO3-379). RADARSAT images were provided by P.W. Vachon from CCRS, and copyright remains CSA. I would like to thank S. Ramos and C. Nava for their support in field measurement activities, and P. Vachon for his comments on the subject of this research. Valuable comments by anonymous reviewers are also acknowledged.

\section{REFERENCES}

Beal, R.C., T.W. Gerling, D.E. Irvine, F.M. Monaldo and D.G. Tiley. - 1986. Spatial variations of ocean wave directional spectra from the Seasat synthetic aperture radar. J. Geophys. 
Res., 91: 2433-2449.

Chapron, B., A.K. Liu, C.Y. Peng and E. Mollo-Christensen. 1995. Higher order spectral and scale analysis of surface wave height. Global Atmos. Ocean Syst., 3: 151-173.

De Valk, C. F. - 1994. A wind and wave data assimilation scheme based on the adjoint technique. In H. Komen (ed.): Dynamics and modelling of ocean waves, pp. 460-468.

Donelan, M.A., W.M. Drennan and A.K. Magnusson. - 1996. Nonstationary analysis of the directional properties of propagating waves. J. Phys. Oceanogr., 26: 1901-1914.

Dunlap, E.M., R.B. Olsen, L. Wilson, S. De Margerie and R. Lalbeharry. - 1998. The effect of assimilating ERS-1 fast delivery wave data into the North Atlantic WAM model. J. Geophys. Res., 103: 7901-7915.

Fu, L.L. and B. Holt. - 1982. Seasat views oceans and sea ice with synthetic aperture radar. Publ. 81-120, Jet Propulsion Laboratory, Pasadena, California.

Hasselmann, S., P. Lionello, and K. Hasselmann. - 1997. An optimal interpolation scheme for the assimilation of spectral wave data. J. Geophys. Res., 102: 15823-15836.

Heimbach, P., S. Hasselmann and H. Hasselmann. - 1998. Statistical analysis and intercomparison of WAM model data with global ERS-1 SAR wave mode spectral retrievals. J. Geophys. Res., 103: 7931-7977.

Hwang, P.A, E.J. Walsh, W.B. Krabill, R.N. Swift, S.S. Manizade, J.F. Scott and M.D. Earle. - 1998. Airborne remote sensing applications to coastal wave research. J. Geophys. Res., 103: 18,791-18,800.

Liu, A.K., C.Y. Peng, B. Chapron, E. Mollo-Christensen and N. Huang. - 1995. Directional and magnitude characteristics of wind stresses over wave groups during SWADE. Global Atmos. Ocean Syst., 3: 175-194.
Luscombe, A.P., I. Ferguson, N. Shepherd, D.G. Zimcik and P. Naraine. - 1993. The RADARSAT synthetic aperture radar development. Can. J. Rem. Sens., 4: 298-310.

McGregor, J.A., E.M. Poulter and M.J. Smith. - 1998. S-band Doppler radar measurements of bathymetry, wave energy fluxes, and dissipation across an offshore bar. J. Geophys. Res., 103: 18,779-18,789.

Mollo-Christensen, E. and A. Ramamonjiarisoa. - 1978. Modeling the presence of wave groups in a random wave field. J. Geophys. Res., 83: 4117-4122.

Ocampo-Torres, F.J. - 1998. Spatial variations of directional wave spectra in coastal waters. Proc. Provision and engineering/operational application of ocean wave data. UNESCO, Paris, 21-25 September 1998, WMO/TD-No. 938, pp 21-30.

Ocampo-Torres F.J., A. Martínez Díaz de León and I.S. Robinson. - 1997. Synergy of ERS radar information and modelled directional wave spectrum to estimate coastal region wave characteristics in the Gulf of Tehuantepec, México. Proc. Int. Seminar on the use and Applications of ERS in Latin American, 25-29 Nov., Viña del Mar, Chile, ESA SP-405, pp. 219-224.

Parashar, S., E. Langham, J. McNally and S. Ahmed. - 1993. RADARSAT mission requirements and concept. Can. J. Rem. Sens., 4: 280-288.

Peng, C.Y., A.K. Liu, B. Chapron, and E. Mollo-Christensen. - 1995. Wavelet analysis of sea surface flux and wave modulation by swell. Global Atmos. Ocean Syst., 3: 195-208.

Shuchman, R.A. and G.A. Meadows. - 1980. Airborne synthetic aperture radar observation of the surf zone conditions. Geophys. Res. Lett., 7, 857-860.

Vachon, P.W., H.E. Krogstad and J.S. Paterson. - 1994. Airborne and spaceborne synthetic aperture radar observations of ocean waves. Atmos. Ocean, 32: 83-112. 